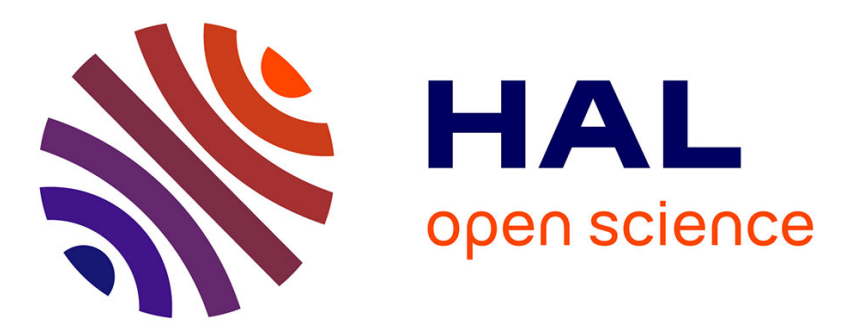

\title{
Stability and structure of adaptive self-organized supramolecular artificial water channels in lipid bilayers
}

A Hardiagon, S Murail, L Huang, M Barboiu, F Sterpone, Marc Baaden

\section{To cite this version:}

A Hardiagon, S Murail, L Huang, M Barboiu, F Sterpone, et al.. Stability and structure of adaptive self-organized supramolecular artificial water channels in lipid bilayers. J.M. Abadie, M., Pinteala, M., Rotaru, A. New Trends in Macromolecular and Supramolecular Chemistry for Biological Applications, Springer, Cham., pp.51-63, 2021, 10.1007/978-3-030-57456-7_3 . hal-02944940

\author{
HAL Id: hal-02944940 \\ https://hal.science/hal-02944940
}

Submitted on 21 Sep 2020

HAL is a multi-disciplinary open access archive for the deposit and dissemination of scientific research documents, whether they are published or not. The documents may come from teaching and research institutions in France or abroad, or from public or private research centers.
L'archive ouverte pluridisciplinaire HAL, est destinée au dépôt et à la diffusion de documents scientifiques de niveau recherche, publiés ou non, émanant des établissements d'enseignement et de recherche français ou étrangers, des laboratoires publics ou privés. 


\title{
Stability and structure of adaptive self-organized supramolecular artificial water channels in lipid bilayers
}

\author{
A. Hardiagon ${ }^{1,2}$, S. Murail ${ }^{3}$, L. Huang ${ }^{4}$, M. Barboiu $^{4}$, F. Sterpone $^{1,2, \#}$, M. Baaden $^{1,2, \#}$ \\ 1) CNRS, Université de Paris, UPR 9080, Laboratoire de Biochimie Théorique, 13 rue Pierre et Marie Curie, F-75005, Paris, France \\ 2) Institut de Biologie Physico-Chimique-Fondation Edmond de Rotschild, PSL Research University, Paris, France
}

3) Université de Paris, CNRS, INSERM, Biologie Fonctionnelle et Adaptative UMR 8251, Computational Modeling of Protein Ligand Interactions U1133, F-75205 Paris, France.

4) Institut Europeen des Membranes, Adaptive Supramolecular Nanosystems Group, Université de Montpellier, ENSCM, CNRS, Place Eugene Bataillon CC047, Montpellier, F- 34095, France

\# co-corresponding authors

\begin{abstract}
Nanopores that efficiently and selectively transport water have been intensively studied at the nanoscale level. A key challenge relates to linking the nanoscale to the compound's macroscopic properties, which are hardly accessible at the smaller scale. Here we numerically investigate the influence of varying the dimensions of a self-assembled Imidazole I-quartet $\left(\mathrm{I}_{4}\right)$ aggregate in lipid bilayers on the water permeation properties of these highly packed water channels. Quantitative transport studies reveal that water pathways in $\mathrm{I}_{4}$ crystal-like packing are not affected by small scaling factors, despite non-uniform contributions between central channels shielded from the bilayer and lateral, exposed channels. The permeation rate computed in simulations overestimates the experimental value by an order of magnitude, yet these in silico properties are very dependent on the force field parameters. The diversity of observed water pathways in such a smallscale in silico experiment yields some insights into modifying the current molecular designs in order to considerably improve water transport in scalable membranes.
\end{abstract}

KEYWORDS : Artificial water channels, self-assembly, molecular dynamics simulations

\section{Introduction}

The quality and purity of available water resources is one of the biggest challenges of our current societies. The increasing water demand has stimulated the development of new technologies such as ultra-purification of water for medical usage or water desalination reverse osmosis. Inspired by recent work on biological systems, new expectations in terms of feasibility and functionality brought researchers towards artificial waterchannel (AWC) systems. Indeed AWCs have the advantage to present a diversity of properties due to their chemical synthesis and they are easily encapsulated in lipids and polymer membranes [1]. Following suggestions about size scaling effects in carbon nanotubes [2,3] and recent studies on monomeric superstructures of AWCs [4], we propose to study the dimensional scalability and the robustness of empirical models using an artificial multimeric channel aggregate.Imidazole Iquartets $\left(\mathrm{I}_{4}\right)$ form a class of artificial waterchannels that experimentally show high selectivity and water conductance [5]. Previous studies have simulated the nanoscopic water transport and the packing of water in similar systems $[5,6]$. We have previously shown that, depending on the morphology of the synthesized compound [7, 8], self-assembled structures in crystalline and amorphous phases account for single water-wire and associated water transport properties.

Experimental evidence mainly comes from stopped-flow experiments in which compounds are assembled in lipid mixtures into monodisperse liposomes of $\sim 100 \mathrm{~nm}$ diameter. This technique employs the use of osmotic gradients to shrink or inflate liposomes. The permeability of the channels embedded in lipids are extracted from the inflation rate probed by light-scattering analysis. 
In order to bridge the gap between experimental measurements occurring at the macroscopic scale and the atomistic description, we propose to study the eventual finite-size effects generated by the boundary conditions chosen for molecular dynamics (MD) simulations of these objects, a method of choice to investigate nano-scale membrane aggregates. We have submitted the selected systems to a series of tests by varying force field parameters to determine their relevance.

We show that growing structures up to $10 \mathrm{~nm}$ long does not lead to pronounced variability in terms of permeation per channel, despite the increase in surface-to-volume ratio. Accordingly, we observe that simulations of larger systems enable us to explore rare permeation events occurring in regions shielded from lipid interactions thanks to an increase in sampling statistics. Furthermore such an approach paves the way to further investigate spatial correlations between adjacent channels in these highly packed structures.

The influence of a range of parameters in the model, such as water molecule representation and force fields for the 14 compounds demonstrate the sensitivity of these models and the complex diversity of water transport mechanisms that arise at such a small spatial scale.

\section{Materials and methods}

All molecular dynamics simulations were performed under periodic boundary conditions with constant pressure. The CHARMM-36 force field [9] was used for lipid molecules and the TIP3P and SPC/E models were chosen [10] for water. To represent S-HC8 molecules and generate their topologies, we used the CHARMM General Force Field [11] together with the ParamChem web service [12]. The GROMACS 2018.7 and 2019.2 softwares were used to run the simulations with all atoms, with an integration time step of $2 \mathrm{fs}$. All bonds were constrained using the LINear Constraint Solver algorithm. Particle mesh Ewald electrostatics was used with a $12 \AA$ cutoff with the Verlet buffer scheme for nonbonded interactions; the neighbor list was updated every 20 steps. Three baths (imidazoles, lipids, and water and ions) were coupled to a temperature of $310 \mathrm{~K}$ using the Nosé-Hoover thermostat with a time constant $t=1 \mathrm{ps}$ and a chain lengh of 4 . Pressure in the $x / y$ dimensions was scaled isotropically with ParrinelloRahman barostat at $1 \mathrm{bar}$, and the $\mathrm{z}$ dimension was coupled independently to a reference pressure of 1 bar, $t=5.0 \mathrm{ps}$, and compressibility of $4.510^{-5} \mathrm{bar}^{-1}$. All systems were minimized for 5000 steps with a steepest descent algorithm and equilibrated for $2 \mathrm{~ns}$, using decreasing position restraints of $1000,400,200,100,40 \mathrm{~kJ} \mathrm{~mol}^{-1} \mathrm{~nm}^{-2}$ on heavy atoms, with the crystal structure as a reference. Production runs were finally computed for 1000 to $2000 \mathrm{~ns}$ without any position restraints.

The previous results we compared to were obtained using initial I-quartet channel patches of $3 \mathrm{~nm}$ width inserted [7]. The packing of the initial patch was based on X-ray structures and the composition of lipids follows the one used experimentally. This composition of the lipid mixture is the same as before with a respective molar ratio 5:4:1 for Chl:PC:PS. The molar ratio between compounds and lipids was previously based on an estimation of the insertion efficiency and initial concentrations inserted [5]. In this new series of simulations, we varied the size of the patch along the two directions of the crystal parallel to the bilayer membrane. The size effect was only investigated in the case of the S-HC8 compound since this structure was the most stable structure of the crystal form observed in previous results. The molar ratio of lipid:compound was multiplied by a factor 2 in simulation sim5, with the same initial patch of circa $3 \mathrm{~nm}$ width. The height of the crystal patch was kept constant and is assumed to be close to the thickness of the lipid bilayer.

All systems have been hydrated with a constant molar ratio of lipid:water equal to 28 . We checked in additional simulations that no other long-range effects on the $z$ axis and through 
periodic boundary conditions occur, by changing this latter molar ratio to 54 . $\mathrm{Na}^{+}$and $\mathrm{Cl}^{-}$ions have been inserted using a Monte-Carlo scheme to neutralize the system and to obtain a concentration of solute equal to $0.15 \mathrm{M}$.

All setups were assembled using the CHARMM-GUI web interface [13] and format files were adapted to the Gromacs software using the online tool services [14].

The molecular dynamics trajectories were visually inspected and analyzed using the VMD software [15]. Analysis of the trajectories for RSMD and permeation calculations were performed using custom scripts for the MDAnalysis library $[16,17]$. The accuracy of the analysis was first tested by varying the sampling time. Fast computation and accuracy were accessed both using $0.1 \mathrm{~ns}$ as a sampling time for the analysis of water permeations. The analysis of pore radius was performed using the HOLE software [18]. Due to large fluctuations of channel components on the lateral sides of the patch, mean pore radius values were relevant only for central channels.

\section{Water Conductance and dimensional scaling}

For our simulations, we computed the average number of permeation events in one direction or water conductance $q_{0}$ (see Table 1). This number is related to the diffusional permeability $p_{d}$ which quantifies the number of water molecules that exited a channel on one side of the membrane once they entered through the opposite side of the membrane [19]. We will further discuss the implications of this specific choice of permeation measurement.

We observed that all systems are stable in the bilayer during 1000 ns simulation, based on their low root mean square deviation from the starting point. There is no major rearrangement of compounds in the membrane, except for very few compounds that move freely within the membrane. A crystal structure arrangement is dominant; we observed very similar water pathways as in previous studies of similar patch configurations (sim1) [7]. In Fig.2 we reported the evolution of water permeation events during the time of each simulation (from 1000 to $2000 \mathrm{~ns}$ ). We observed that an equilibrium regime is reached on a range of time between 500 and 1000 ns depending on the simulation.

All simulations were performed under equilibrium conditions, therefore we expect that the net flow through the channels is null on average after a certain time. Indeed, we observed that the net flow cancellation coincides with the convergence of permeation rates to a constant value. Regarding these observations, we extracted water conduction using water molecule trajectories taken above a time offset considered as equilibration period. We combine linear regression and a bootstrap method to compute estimates of water conductance and their confidence intervals [20]. We computed conductance values reported in Table 1 using the following relation :

$$
q_{0}=\frac{N_{\text {perm }}}{2 N_{c}}
$$

where $N_{\text {perm }}$ relates to the number of permeation events per second (in both directions), $N_{c}$ relates to the effective number of channels. We consider a "permeation event" as a complete transport of a water molecule through the channel delimited by the mean position of phosphorus atoms in the lipid bilayer in both leaflets. The number of effective channels in our simulations was estimated by visual examination. Thereby we defined an effective channel as an 14- (or lipid-)bounded area within which water wires are located; the "effective" attribute characterizing channels delimited by both compound molecules and hydrophobic tails of lipid molecules that allow water to cross the membrane. Despite basing the structure of the central channel on X-ray data, the behavior of the channels exposed to lipids was first unclear to us. As most of the 
permeation events occur in these regions, we tested two different setups : one patch with the lateral, exposed channels defined by exactly four imidazole molecules and one patch with exposed lateral channels defined by three imidazole molecules, see Fig.1 a)b), the latter setup was already used in previous studies [7]. Both configurations remain stable during $1000 \mathrm{~ns}$ and we notice higher thermal fluctuations for the imidazole column in the first setup, resulting in only one lateral-exposed channel and a reduced number of effective channels, see Fig. 1.

Interestingly, in the second regime we observed that the normalized number of permeation events grows with a similar rate independently of the size of either the membrane or the patch. In the next section, we further discuss different considerations about the water pathways in order to better understand these results.

In previous results, simulations were performed using a larger lateral pressure in $x / y$ directions (10atm) compared to normal pressure (1atm). Our motivation for this choice was to compensate for the relatively small size of the aggregates. Indeed, it has been shown that small compounds tend to remain in crystal form under higher lateral pressure. In the present simulations, we did not need to increase lateral pressure since the crystal form was already stable at 1atm. Some preliminary simulations have been performed at higher lateral pressure (10 atm) but no significant changes in both stability and permeations were observed. One of the simulation outcomes (sim5) with a larger lipid membrane indicates that the number of lipids does not play a role in the overall stability since permeation pathways are very similar to observations in sim1. We believe that the coupling between chiral crystal and induced chiral water single-wire is enforcing the stability of this chiral structure [6], resulting in the observed robustness of the permeation mechanism. At this stage, we plan to perform a more detailed analysis of the contribution of each Van der Waals and Coulomb interaction between compound/compound, compound/lipid and compound/water pairs. This breakdown will allow us to understand the key mechanism for stability and permeation in these structures.

\section{Permeation pathways : lateral versus central}

In all simulations we observed a much larger number of permeations in lateral channels compared to central channels. Due to the multimeric nature of the patch, channels were identified based on geometrical aspects given the position of each water molecule permeating in the half-time of its course through the membrane. Considering the array-like alignment of channels in one direction in the membrane plane, we considered as "lateral channel" either an exposed channel with walls sharing lipid atoms and compound atoms, or the first channel with walls made by compound molecules. This choice was motivated by observations of water molecules jumping laterally from one channel to another in this region. Note that the average duration of permeation events, despite a broad range of deviations is always bigger in central channels (10-500ns) than in lateral channels (1-50 ns). These values are reported qualitatively here, despite the lack of statistics on these timescales to distinguish water permeation mechanisms between central and lateral channels.

Following the last two columns of Table 1, we noticed that systems with bigger size patches display more central permeation events. Interestingly, the permeation in central channels is not equally distributed among the central channels and only a few of them are activated (see Fig. 3). An analysis of the pore radius was performed on a trajectory obtained from sim4 and was correlated with the permeation events. Over 10 central channels, only two pairs of adjacent channels remain active in the second part of the simulation (channels 3,4 and 7,8 ). For these channels, the mean pore radius is $1.36 \pm 0.1 \AA$ and for inactive channels the mean pore radius is $1.31 \pm 0.1 \AA$ (see Fig.3). Thus, central permeation events are correlated to fluctuations of the pore size. 
In several simulations not reported here, we observed an increase of central channel radius up to $1.5 \AA$ which is accompanied by a much faster transport of water with a change of conformation of the water packing inside a channel. These results suggest that the patch could undergo some structural transitions at room temperature. However, we want to warn the reader that further investigation of these dynamical processes is still to be investigated. Indeed the statistics of central permeation events at these timescales remain too low to ensure the system to be in a stationary state, and therefore compute a stationary permeation rate.

In order to characterize the permeation pathways, we also computed the unidirectional flow for both directions along the $\mathrm{z}$ axis on the time window where the absolute flow is constant. We observed the water flow to be almost symmetric for all lateral channels. Moreover we noticed that central channels in large systems could maintain a positive net flow in the active channel during more than $400 \mathrm{~ns}$ (see Fig. 3). Still, there is no evidence that water flow in one direction could be energetically favored in this equilibrium-MD simulation. To confirm this hypothesis, it could be interesting to extend the simulations considering its limitations due to the small amount of statistics for central channels in the current study.

\section{Discussion on the model}

We showed that for the scales investigated the overall water conductance scales linearly with the size of the patch. At first sight, if we suppose that all channels of the patch contribute independently to the overall water flow, these results seem natural. However a detailed analysis of individual channel permeation has revealed that lateral channels contribute significantly more permeation events than central channels. If we consider simulation sim4 (see Figure 1), the setup has the same number of lateral channels as in sim1, but with almost the double number of central channels. First, we were expecting that the water conductance will scale with the number of lateral channels, but simulations show that it scales with the total number of channels. One hypothesis to understand these results is to suggest that permeation in these crystal-like structures is a collective phenomenon between adjacent channels. This vision is supported by the comparison between simulations $\operatorname{sim} 1$ and $\operatorname{sim} 2$ where the local change of the lateral channel conformation does not seem to disturb overall water conductance. So, in order to understand the mechanism of water flow at larger scales, one has to consider the spatial correlations of such crystal-like molecular assemblies.

As shown in Fig.1, the choice of the water model has a significant impact on the permeation rate. Water conduction is twice stronger using the TIP3P water model compared to the SPC/E model given all other setup features being equal. It is well-known that water transport in confined geometries is strongly dependent on hydrogen bonds that water molecules form within their close environment [21, 22]. Yet the number of hydrogen-bonds in S-HC8 channels is very close to the number of hydrogen bonds in the bulk. Hence we expect that the diffusion in the channels at equilibrium will be affected correspondingly to the bulk diffusion. Indeed, as shown in Table 2, the ratio between self-diffusion coefficients between TIP3P and SPC/E model is 2.1 which is consistent with our results. Moreover, as the SPC/E water model is more faithful to real data concerning transport properties, we keep in mind that almost all simulations performed here with the TIP3P model would overestimate transport properties.

In order to test the sensitivity of the model coefficients, we compared the water conductance in two similar setups varying force field parameters for the imidazole compounds using either CGenFF [23] or GAFF [24]. Results in Fig.1 show that permeability is more than three times larger when using the GAFF force field. Using GAFF parameters, we noticed the crystal patch to be less flexible under thermal fluctuations; almost no imidazole molecules are moving freely in the lipid bilayer. We expect that GAFF parameters reinforce long-range electrostatics interactions resulting in even more stable crystal-like patches embedded in the 
lipid bilayer. This freezing effect of the surrounding architecture could contribute to increase water conductance both in lateral and central channels. However, further considerations about energetics and experimental data is under investigation to validate and study the influence of both LJ coefficients and partial charges of these two models.

Furthermore, experimental data is required to assess the validity of the modeled aggregates. Despite the estimation from experimental results of the molar ratio between lipids and compound molecules, the average size of these membrane-embedded objects actually remains unknown. Two scenarios arise at this stage, one assuming the existence of small patches with large surface-to-volume-ratio effect and therefore a particular role of the interaction between lipids and the compound patch on permeation rate, the other scenario assuming the presence of large crystal patches where surface-to-volume-ratio effects are negligible. In order to validate the second scenario, we would need above-mentioned experimental data. Furthermore a coarse-grain approach could be adopted to simulate larger systems, however the forcefield issue then becomes even more stringent as all relevant physico-chemical properties of the imidazole compounds will have to be captured in the simplified representation. Somehow we would have to find a compromise between the loss of atomistic description for water dynamics and the gain in the amount of statistics.

Although the size of the embedded system does not seem to play a significant role in the permeation rate in our simulations, we keep in mind that it essentially concerns the hypothesis based on crystal configuration at small timescale. The major difficulty in reproducing the experimental conditions relates to the unknown average size and distribution of compound assemblies embedded in the liposomes, the radius of which is distributed around $100 \mathrm{~nm}$. In our simulations, the patch size does not exceed $10 \mathrm{~nm}$ and timescales accessible to all-atoms descriptions are bounded to a few microseconds. Given the small values of the scaling factor we used in this study, we do not exclude some important surface-to-volume effects when we scale the system up to experimental ones. For the time being, we cannot resolve the question of long-term events such as the rearrangement of the structure inside the membrane without validating a proper force field. We already know from previous results related to other compounds [7] that disordered aggregates may also display high water conductance and ion selectivity but these structures have shown limited robustness under application of the current force field. This observation emphasizes the difficulty to validate the force field choice at these small timescales.

Regarding the test series we performed on the S-HC8 compound, we can minimize the actual mismatch between experimental results in stopped-flow experiments and our simulations where we found a water conductance of one to two orders of magnitude larger than the experimental value. Indeed, the choice of the model needs to be understood in order to correctly reproduce tendencies of the experiments. Moreover, the current studies were only tackling the stability and permeation mechanisms at equilibrium. Yet structural changes of such systems composed by a large number of interacting molecules has to be challenged under nonequilibrium conditions such as osmotic gradients or hydrostatic pressure [25, 26]. Such considerations as a comparison between diffusional and osmotic single-channel permeabilities need to be clarified on these systems, in order to enlighten experimental observations in real systems. Although these dynamical aspects have been intensively studied in carbon nanotubes and biological channels [21], a long road to understand stability and water permeation mechanisms in molecular self-assemblies remains.

\section{Conclusion and perspectives}

By varying the size of the patch of crystal-like assemblies of $\mathrm{I}_{4}$ channels using MD simulations, we found that permeation rate scales with the total of number of channels. Large 
surface-to-volume-ratio in these in silico model systems could induce an overestimation of the permeation rate in contrast to experiments on liposome substrates. Surprisingly, finite-size effects do not seem to be ruled out by the particular morphology of the more active channels. These findings suggest that collective effects that imply long-range interactions between compounds and lipid/compound interfacial energies have to be carefully investigated. Indeed, the scaling of these seed structures to a larger scale remains a challenge given the number of uncertainties related to the phase state of large self-assemblies in lipid bilayers. Although X-ray data provide a crystalline structure that displays permeation properties, we showed that the force field parameters that describe interactions between $\mathrm{I}_{4}$ compounds is determinant to allow stability within the lipid bilayer and therefore control the permeation rate. Flexibility and mutual effects between hydrophobic and hydrophilic regions in these packed channels have shown to be a major asset to increase single-channel permeability. Simulations will play an important role in elaborating future designs of self-assembled compounds with improved properties. Previous and present studies rely on a static approach, therefore out-of-equilibrium simulations will confirm and complete the current scenario. Furthermore, Van der Waals interactions and electrostatic interactions will be investigated to calibrate the force field with respect to experimental data. At last, larger scales will be assessed by coarse-grained approaches [27, 28] in order to study the feasibility of scalable membrane constructs linking to the macro-scale [29, 30].

\section{Acknowledgements}

This work was conducted within the ANR-15-CE29-0009 DYNAFUN (Mihail Barboiu) and ANR-18-CE06-0004 WATERCHANNELS (Fabio Sterpone) projects. The study was further supported by the "Initiative d'Excellence" program from the French State (Grant "DYNAMO", ANR-11-LABX-0011 and grant "CACSICE", ANR-11-EQPX-0008). A.H. and M.B. thank Sesame lle-de-France for co-funding the display wall used for data analysis. Computational work was performed using HPC resources from GENCI-CINES (grant number A0070701714) to Marc Baaden. This publication received support from the European Union's Horizon 2020 research and innovation programme under Grant No. 667387 WIDESPREAD 2-2014 SupraChem Lab. 

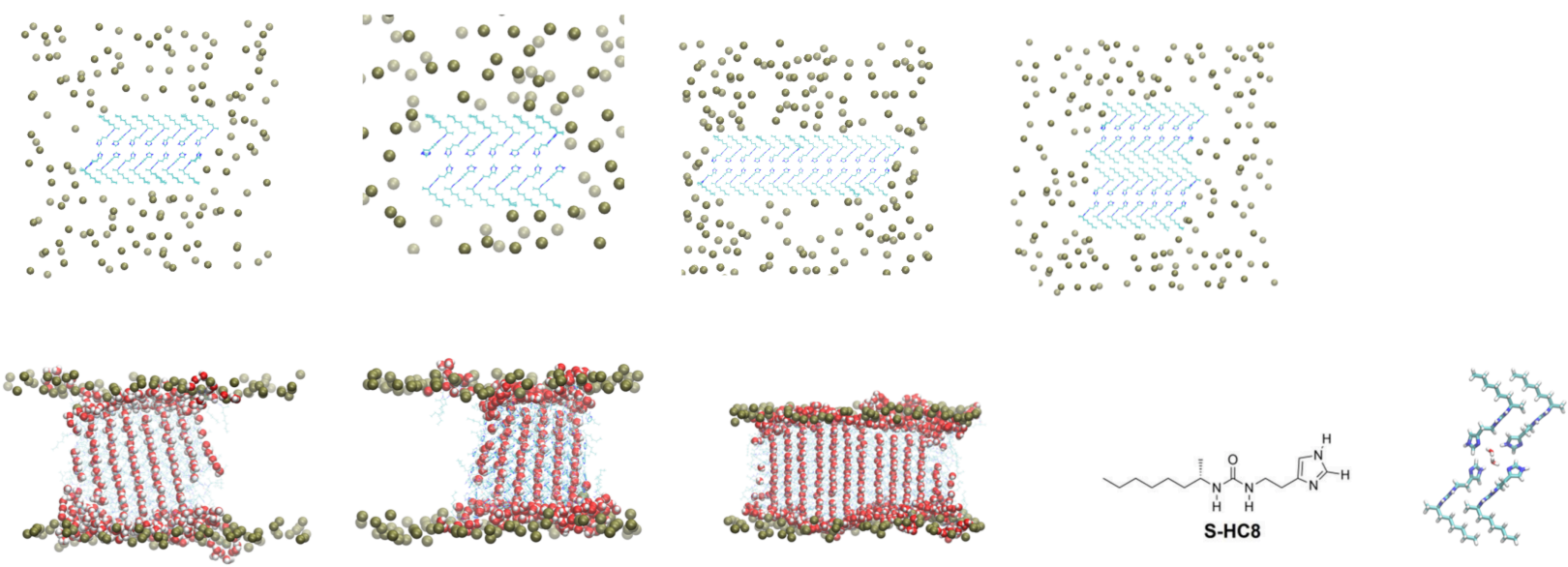

$$
\mathrm{N}_{\text {eff }}=8
$$

$\mathrm{N}_{\text {eff }}=6$

$\mathrm{N}_{\mathrm{eff}}=14$

Figure 1 : Initial aggregation setups of the S-HC8 compound (see panels $h$ and i) used for molecular dynamics simulations. Top view (a)sim1 (b)sim2 (c)sim3 (d)sim4 . Lateral view (e)sim1 (f) sim2 (g)sim3. Only molecules of the 14 compounds and the phosphorus atoms of the lipids are represented in the upper panels; water molecules close to polar heads of the imidazole compound are added in the bottom panels. All other molecules are omitted in the pictures for clarity. Below each bottom panel, the estimated number of effective water channels is shown. 

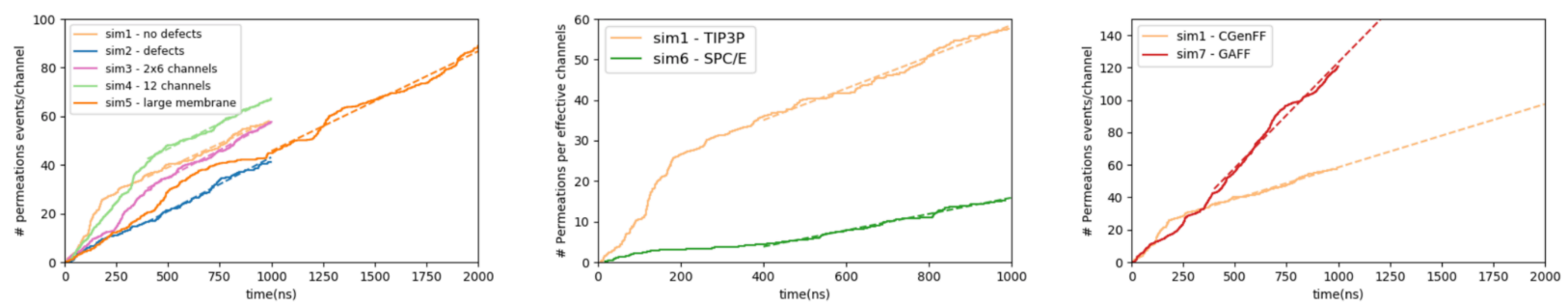

Figure 2 : Time series of number of permeation events normalized over the total number of pre-formed imidazole 14 channels in the membrane. The permeation rates are grouped together with respect to several varying factors: size of the patch and lipid/compound ratio (a), water model (b) and force field parameters (c). Achieving a constant permeation rate after an initial time offset provides a simple measure that the system reaches a converged transport state. Water conductance values associated to each simulation are reported in Table 1. 

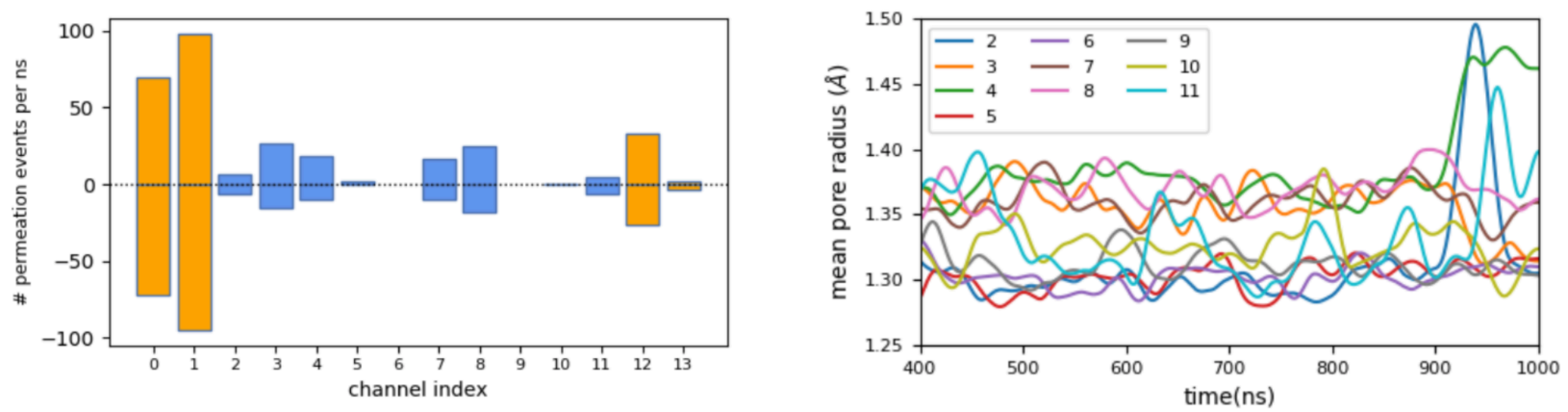

Figure 3 : (a) water conductance per channel (12 aligned channels) during the time window 400-1000ns. Channel indices follow the spatial order, the channels 0 and 13 are effective channels delimited by both lipids and S-HC 8 molecules. Lateral channels are colored in orange and central channels are colored in blue. Positive values account for water molecules entering the lower leaflet and exiting the channel on the upper leaflet, and negative values are defined accordingly. (b) Time series of mean pore radius for each labeled central channel. Lateral mean pore radius for lateral channels was not represented, indeed higher fluctuations of channel walls reduces considerably the accuracy of this measure. Only the time window corresponding to a constant water flow is considered. For convenience, data is averaged on a time window of 50 ns. 
Table 1 : Water conductance values reported for the whole set of simulations. Some results from previous studies and from previous experimental measurements are mentioned for comparison. Water conductance values are computed using number of permeation events in a time window normalized by the number of effective channels in the pre-formed crystal patch. The last two columns account for the distribution of events between lateral and central channel as defined in the article text.

\begin{tabular}{|c|c|c|c|c|c|c|c|c|}
\hline \multirow{3}{*}{$\begin{array}{l}\text { Simulation } \\
\text { acronym }\end{array}$} & \multirow[t]{3}{*}{ System description } & \multirow{3}{*}{$\begin{array}{l}\text { Force } \\
\text { Field }\end{array}$} & \multirow{3}{*}{$\begin{array}{l}\text { Water } \\
\text { Model }\end{array}$} & \multirow{3}{*}{$\begin{array}{c}\text { Permeation } \\
\text { regime }\end{array}$} & \multicolumn{4}{|c|}{ Conductance } \\
\hline & & & & & \multicolumn{2}{|c|}{$\mathrm{x} 10^{6} \mathrm{H} 20 / \mathrm{s} /$ channel } & \multirow[t]{2}{*}{ lateral } & \multirow[t]{2}{*}{ central } \\
\hline & & & & & $q_{0}$ & std & & \\
\hline & AQP1 & - & - & - & $\sim 10^{3}$ & - & - & - \\
\hline & S-HC8 - Exp (7) & - & - & osmotic & 1,4 & 0,1 & - & - \\
\hline $\operatorname{sim} 1$ & S-HC8 - 6 channels - no defects & CgenFF & TIP3P & diffusive & 19.5 & 0.2 & $99 \%$ & $1 \%$ \\
\hline $\operatorname{sim} 2$ & $\begin{array}{l}\text { S-HC8 - } 6 \text { channels - with } \\
\text { defects }\end{array}$ & CgenFF & TIP3P & diffusive & 22.2 & 0.2 & $99 \%$ & $1 \%$ \\
\hline $\operatorname{sim} 3$ & S-HC8 - 2x6 channels & CgenFF & TIP3P & diffusive & 22.5 & 0.1 & $82 \%$ & $18 \%$ \\
\hline $\operatorname{sim} 4$ & SHC8 - 12 channels & CgenFF & TIP3P & diffusive & 20.8 & 0.1 & $72 \%$ & $28 \%$ \\
\hline $\operatorname{sim} 5$ & $\begin{array}{l}\text { S-HC8 - } 6 \text { channels - large } \\
\text { membrane }\end{array}$ & CgenFF & TIP3P & diffusive & 20.5 & 0.1 & $98 \%$ & $2 \%$ \\
\hline $\operatorname{sim} 6$ & S-HC8 - 6 channels & CgenFF & SPC/E & diffusive & 10.1 & 0.1 & $76 \%$ & $14 \%$ \\
\hline $\operatorname{sim} 7$ & S-HC8 - 6 channels & GAFF & TIP3P & diffusive & 65.0 & 0.3 & $78 \%$ & $28 \%$ \\
\hline
\end{tabular}


Table 2 : Self-diffusion of water in the bulk calculated for two water models and experimental reference value [31] \begin{tabular}{|l|l}
\hline Water Model & Self-diffusion $\left(10^{-5} \mathrm{~cm}^{2} / \mathrm{s}\right)$
\end{tabular}

\begin{tabular}{|c|c|}
\hline experimental & 2.30 \\
\hline TIP3P & 5.19 \\
\hline SPC/E & 2.49 \\
\hline
\end{tabular}




\section{References}

1. Tan Z, Chen S, Peng X, et al (2018) Polyamide membranes with nanoscale Turing structures for water purification. Science 360:518-521. https://doi.org/10.1126/science.aar6308

2. Garate J-A, English NJ, MacEIroy JMD (2009) Static and alternating electric field and distance-dependent effects on carbon nanotube-assisted water self-diffusion across lipid membranes. J Chem Phys 131:114508. https://doi.org/10.1063/1.3227042

3. Köfinger J, Hummer G, Dellago C (2008) Macroscopically ordered water in nanopores. Proc Natl Acad Sci 105:13218-13222. https://doi.org/10.1073/pnas.0801448105

4. Noy A, Wanunu M (2020) A new type of artificial water channels. Nat Nanotechnol 15:9-10. https://doi.org/10.1038/s41565-019-0617-5

5. Licsandru E, Kocsis I, Shen Y, et al (2016) Salt-Excluding Artificial Water Channels Exhibiting Enhanced Dipolar Water and Proton Translocation. J Am Chem Soc 138:5403-5409. https://doi.org/10.1021/jacs.6b01811

6. Kocsis I, Sorci M, Vanselous $\mathrm{H}$, et al (2018) Oriented chiral water wires in artificial transmembrane channels. Sci Adv 4:eaao5603. https://doi.org/10.1126/sciadv.aao5603

7. Murail S, Vasiliu T, Neamtu A, et al (2018) Water permeation across artificial I-quartet membrane channels: from structure to disorder. Faraday Discuss 209:125-148.

https://doi.org/10.1039/C8FD00046H

8. Sun Z, Kocsis I, Li Y, et al (2018) Imidazole derivatives as artificial water channel buildingblocks: structural design influence on water permeability. Faraday Discuss 209:113-124. https://doi.org/10.1039/C8FD00024G

9. Klauda JB, Venable RM, Freites JA, et al (2010) Update of the CHARMM All-Atom Additive Force Field for Lipids: Validation on Six Lipid Types. J Phys Chem B 114:7830-7843. https://doi.org/10.1021/jp101759q

10. Jorgensen WL, Chandrasekhar J, Madura JD, et al (1983) Comparison of simple potential functions for simulating liquid water. J Chem Phys 79:926-935.

https://doi.org/10.1063/1.445869

11. Vanommeslaeghe K, MacKerell AD (2012) Automation of the CHARMM General Force Field (CGenFF) I: Bond Perception and Atom Typing. J Chem Inf Model 52:3144-3154.

https://doi.org/10.1021/ci300363c

12. Vanommeslaeghe K, Raman EP, MacKerell AD (2012) Automation of the CHARMM General Force Field (CGenFF) II: Assignment of Bonded Parameters and Partial Atomic Charges. J Chem Inf Model 52:3155-3168. https://doi.org/10.1021/ci3003649

13. Jo S, Kim T, Iyer VG, Im W (2008) CHARMM-GUI: A web-based graphical user interface for CHARMM. J Comput Chem 29:1859-1865. https://doi.org/10.1002/jcc.20945

14. Lee J, Cheng X, Swails JM, et al (2016) CHARMM-GUI Input Generator for NAMD, GROMACS, AMBER, OpenMM, and CHARMM/OpenMM Simulations Using the CHARMM36 Additive Force Field. J Chem Theory Comput 12:405-413.

https://doi.org/10.1021/acs.jctc.5b00935 
15. Humphrey W, Dalke A, Schulten K (1996) VMD: Visual molecular dynamics. J Mol Graph 14:33-38. https://doi.org/10.1016/0263-7855(96)00018-5

16. Gowers RJ, Linke M, Barnoud J, et al (2016) MDAnalysis: A Python Package for the Rapid Analysis of Molecular Dynamics Simulations. Proc 15th Python Sci Conf 98-105.

https://doi.org/10.25080/Majora-629e541a-00e

17. Michaud Agrawal N, Denning EJ, Woolf TB, Beckstein O (2011) MDAnalysis: A toolkit for the analysis of molecular dynamics simulations. J Comput Chem 32:2319-2327.

https://doi.org/10.1002/jcc.21787

18. Smart OS, Neduvelil JG, Wang X, et al (1996) HOLE: A program for the analysis of the pore dimensions of ion channel structural models. J Mol Graph 14:354-360.

https://doi.org/10.1016/S0263-7855(97)00009-X

19. Zhu F, Tajkhorshid E, Schulten K (2004) Theory and simulation of water permeation in aquaporin-1. Biophys J 86:50-57. https://doi.org/10.1016/S0006-3495(04)74082-5

20. Haslwanter T (2016) Linear Regression Models. In: Haslwanter T (ed) An Introduction to Statistics with Python: With Applications in the Life Sciences. Springer International Publishing, Cham, pp 183-220

21. Horner A, Zocher F, Preiner J, et al (2015) The mobility of single-file water molecules is governed by the number of $\mathrm{H}$-bonds they may form with channel-lining residues. Sci Adv 1:e1400083. https://doi.org/10.1126/sciadv.1400083

22. Alexiadis A, Kassinos S (2008) Self-diffusivity, hydrogen bonding and density of different water models in carbon nanotubes. Mol Simul 34:671-678.

https://doi.org/10.1080/08927020802073057

23. Caleman C, van Maaren PJ, Hong M, et al (2012) Force Field Benchmark of Organic Liquids: Density, Enthalpy of Vaporization, Heat Capacities, Surface Tension, Isothermal Compressibility, Volumetric Expansion Coefficient, and Dielectric Constant. J Chem Theory Comput 8:61-74. https://doi.org/10.1021/ct200731v

24. Wang J, Wolf RM, Caldwell JW, et al (2004) Development and testing of a general amber force field. J Comput Chem 25:1157-1174. https://doi.org/10.1002/jcc.20035

25. Kalra A, Garde S, Hummer G (2003) Osmotic water transport through carbon nanotube membranes. Proc Natl Acad Sci U S A 100:10175-80.

https://doi.org/10.1073/pnas.1633354100

26. Yoshida $\mathrm{H}$, Marbach S, Bocquet $\mathrm{L}$ (2017) Osmotic and diffusio-osmotic flow generation at high solute concentration. II. Molecular dynamics simulations. J Chem Phys 146:194702. https://doi.org/10.1063/1.4981794

27. Chan H, Král P (2018) Nanoparticles Self-Assembly within Lipid Bilayers. ACS Omega 3:10631-10637. https://doi.org/10.1021/acsomega.8b01445

28. F. Brandner A, Timr S, Melchionna S, et al (2019) Modelling lipid systems in fluid with Lattice Boltzmann Molecular Dynamics simulations and hydrodynamics. Sci Rep 9:16450. https://doi.org/10.1038/s41598-019-52760-y 
29. Kumar M, Habel JEO, Shen Y, et al (2012) High-Density Reconstitution of Functional Water Channels into Vesicular and Planar Block Copolymer Membranes. J Am Chem Soc 134:1863118637. https://doi.org/10.1021/ja304721r

30. Shen Y, Song W, Barden DR, et al (2018) Achieving high permeability and enhanced selectivity for Angstrom-scale separations using artificial water channel membranes. Nat Commun 9:1-11. https://doi.org/10.1038/s41467-018-04604-y

31. Mahoney MW, Jorgensen WL (2000) Diffusion constant of the TIP5P model of liquid water.

J Chem Phys 114:363-366. https://doi.org/10.1063/1.1329346 